\title{
Efeito das propriedades dos esmaltes e engobes sobre a curvatura de revestimentos cerâmicos
}

\section{(Effect of glaze and engobe properties on the curvature of ceramic tiles)}

\author{
M. Dal Bóo ${ }^{1,2}$, F. G. Melchiades ${ }^{2}$, A. O. Boschi ${ }^{2}$, D. Hotza' \\ ${ }^{1}$ Programa de Pós-Graduação em Ciência e Engenharia de Materiais - PGMAT, Universidade Federal de Santa \\ Catarina UFSC, Florianópolis, SC 88040-900 \\ ${ }^{2}$ Laboratório de Revestimentos Cerâmicos - LaRC, Departamento de Engenharia de Materiais, Universidade \\ Federal de S. Carlos, Rod. Washington Luiz, km 235, C. P. 676, S. Carlos, SP 13565-905 \\ marcelodalbo@hotmail.com
}

\begin{abstract}
Resumo
As interações entre suporte-esmalte e suporte-engobe foram estudadas separadamente frente à curvatura central de revestimentos cerâmicos. Formularam-se duas amostras de esmaltes e quatro amostras de engobes cerâmicos, as quais foram aplicadas sobre um determinado tipo de suporte cerâmico com classificação dentro do grupo B-IIb, pela norma ABNT NBR 13.818/1997 - ISO 13006. Foram mensuradas características como "coeficiente de expansão térmica linear" e "diferença de retração entre suporte-engobe". Foi proposto um método para a avaliação da interação entre o suporte-esmalte e suporte-engobe. Os resultados evidenciaram que, para a interação suporte-esmalte o método de sobreposição de curvas dilatométricas foi aplicado; para a interação suporte-engobe, realizando um teste estatístico com planejamento fatorial $2^{2}$, evidenciou-se que a variável "diferença de retração linear" entre o suporte e o engobe é a variável significativa para descrever a curvatura central de revestimentos cerâmicos.
\end{abstract}

Palavras-chave: curvatura, engobe, esmalte, revestimentos cerâmicos.

\begin{abstract}
The interaction between support-glaze and support-engobe were studied separately regarding the central curvature of ceramic tiles. Were formulated two glazes samples and four engobes samples, these were applied on a particular type of ceramic support with classification in the group B-IIb, by Norma ABNT NBR 13.818/1997 - ISO 13006. Some characteristics were measured as "Thermal expansion coefficient" and "Shrinkage difference between support-engobe". A method was suggested $d$ for evaluation of interaction between support-engobes and support-glazes. The results showed that, for interaction support-glazes the superposition of dilatometric curves method is well implemented; for interaction support-engobe, a statistical test was conducted with factorial design $2^{2}$, indicating that the variable "Shrinkage difference between support-engobe" is the variable significant to describe the central curvature of ceramic tiles.
\end{abstract}

Keywords: curvature, engobe, glaze, ceramic tiles.

\section{INTRODUCÃO}

Uma das qualidades mais apreciadas nas placas cerâmicas é a sua planaridade. Esta característica é um requisito indispensável para estes produtos atualmente, cuja finalidade é revestir paredes (revestimentos) e solos (pavimentos) melhorando sua qualidade higiênica e estética. Não obstante, durante o processo de fabricação de placas cerâmicas existem vários fatores que podem alterar sua forma plana. especialmente quando se trata de peças esmaltadas conformadas por prensagem seguindo um processo produtivo típico de monoqueima [1].

Os revestimentos cerâmicos produzidos atualmente são compostos geralmente por três camadas distintas: o suporte cerâmico, o engobe e o esmalte. Como pode ser observado na Fig. 1. Estas camadas possuem composição química diferente e conseqüentemente propriedades físicas e químicas distintas. Estudos anteriores [2-11] relatam que, além das variáveis de queima, as características dessas camadas têm grande influência sobre a planaridade de revestimentos cerâmicos. A sinterização de revestimentos cerâmicos provoca uma modificação fundamental em suas propriedades, resultando em um material com alta dureza e apreciável resistência mecânica, a etapa de queima de produtos cerâmicos é considerada uma das etapas mais importantes do processo. Durante a sinterização podem se desenvolver vários defeitos provenientes de outras etapas do processo produtivo como a moagem, conformação, secagem e esmaltação. É após esta etapa que a curvatura de revestimentos cerâmicos é evidenciada [2].

Para estudar a evolução da curvatura das peças cerâmicas durante o tratamento térmico não é suficiente somente conhecer os gradientes de temperatura no interior da peça, mas também é necessário conhecer a relação 


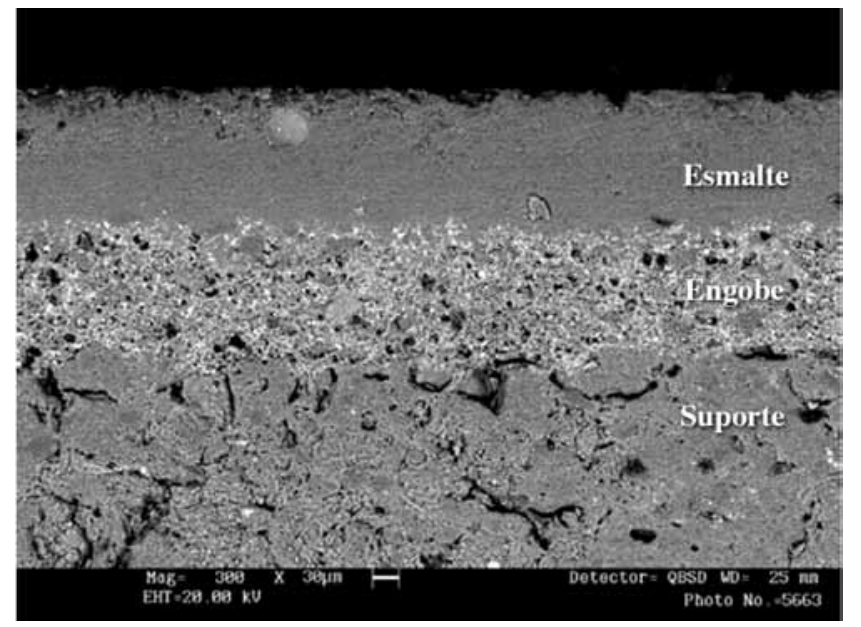

Figura 1: Imagem ilustrativa, obtida por microscopia eletrônica de varredura, da seção transversal de um revestimento cerâmico com visualização das camadas que o compõem [11].

[Figure 1: Transversal section of a ceramic tile obtained by SEM showing the layers that compose it [11].]

que existe entre as tensões geradas por este gradiente de temperatura e deformações; portanto, necessita-se conhecer as propriedades mecânicas das camadas integrantes do revestimento cerâmico [2]. Estas propriedades mecânicas estão definidas pelas leis de comportamento dos materiais, que são relações entre as tensões e deformações resultantes em um ponto de um material.

Recentemente estudos realizados com microscópio óptico de calefação viabilizaram a realização de estudos da curvatura de revestimentos em um determinado ciclo térmico [12], permitindo a realização de estudos de algumas características das camadas que compõem o revestimento cerâmico quanto à curvatura.

São inúmeras as variáveis que têm influência na planaridade de revestimentos cerâmicos, dentre elas podemos citar: coeficiente de expansão térmica linear, retração linear de queima, módulo de elasticidade (Módulo de Young), temperatura de amolecimento de esmaltes, temperatura de transição vítrea de esmaltes, espessura das camadas que compõem o revestimento cerâmico, tamanho da peça cerâmica, além do já comentado ciclo de queima [9]. Assim o estudo do fenômeno de curvatura de revestimentos cerâmicos é um tema complexo, porém com um vasto campo a ser explorado.

O presente trabalho apresenta um estudo relacionando características importantes como a retração linear de queima, o coeficiente de expansão térmica linear e a temperatura de acoplamento entre esmaltes e suportes com a curvatura central de revestimentos cerâmicos em nível de laboratório.

Vários esmaltes cerâmicos, durante o tratamento térmico de sinterização, apresentam características muito similares as do vidro. Uma definição de vidro que pode ser encontrada na bibliografia, é de um líquido de altíssima viscosidade sub-resfriado. Na temperatura ambiente possui aparência de sólido, devido a sua rigidez mecânica, dada pela sua elevada viscosidade. Neste caso não podem ser considerados sólidos, já que possuem sua estrutura amorfa, carecendo portanto de uma estrutura cristalina, que caracteriza e define o estado sólido [13].

\section{Esmaltes cerâmicos}

Os esmaltes cerâmicos cristalinos possuem características semelhantes a um vidro, possuindo portanto uma temperatura de transição vítrea. Para materiais vítreos, o volume decresce continuamente com a redução da temperatura; ocorre um pequeno decréscimo na inclinação da curva o qual é chamado temperatura de transição vítrea, $\mathrm{Tg}$; ilustrado na Fig. 2. Abaixo desta temperatura, o material é considerado como sendo um vidro; acima dela, o material é primeiro um líquido super-resfriado e depois um líquido (no sentido crescente da temperatura) [14].

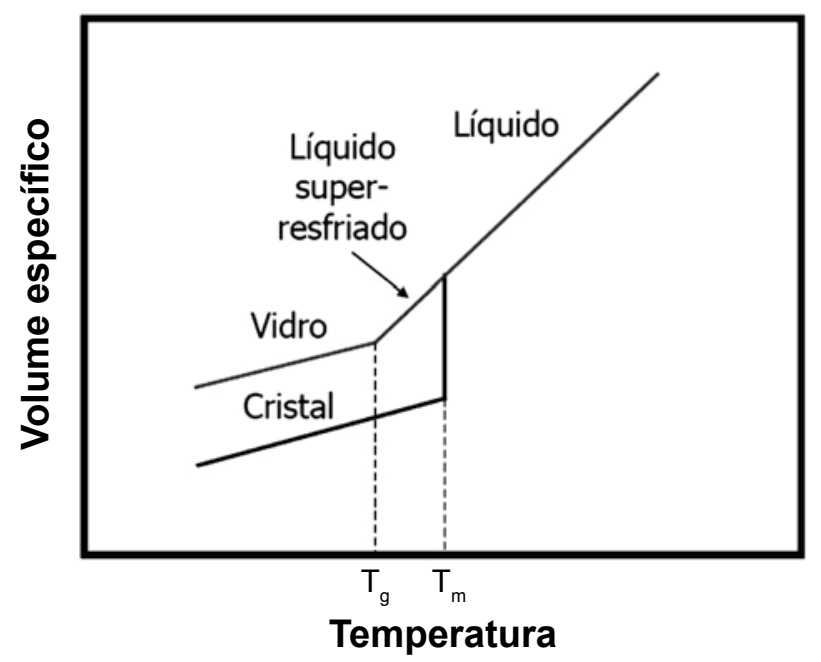

Figura 2: Contraste do comportamento do volume específico versus temperatura de materiais cristalinos e não cristalinos [14].

[Figure 2: Contrast of specific volume versus temperature behavior of crystalline and noncrystalline materials [14].]

Grande parte dos estudos existentes na literatura [8-10, $13,17]$ menciona que a temperatura de acoplamento $\left(\mathrm{T}_{\mathrm{a}}\right)$ entre o esmalte e o suporte cerâmico é considerada como sendo a média entre a temperatura de transição vítrea $\left(\mathrm{T}_{\mathrm{g}}\right)$ e a temperatura de amolecimento (melting point $-\mathrm{T}_{\mathrm{m}}$ ), como ilustrado na equação A:

$$
\mathrm{T}_{\mathrm{a}}=\frac{\mathrm{T}_{\mathrm{g}}+\mathrm{T}_{\mathrm{m}}}{2}
$$

Estudos do comportamento do acoplamento entre esmaltes e suportes cerâmicos têm sido bastante explorados por autores [8-10, 13]. Mostra-se que, somente as leis de comportamento mecânico elástico são suficientes para avaliar este tipo de interação. Descreve-se bem o comportamento da flecha de curvatura (D) de revestimentos cerâmicos, com a equação de Timoshenko; levando-se se em consideração somente a interação esmalte-suporte [9]. A equação B ilustra matematicamente a relação de Timoshenko para a flecha de curvatura (D). 


$$
\mathrm{D}=\frac{\mathrm{L}^{2} \mathrm{~K}_{\mathrm{R}} \Delta \mathrm{C}}{8 \mathrm{~h}}
$$

na qual $\mathrm{D}=$ flecha de curvatura central $(\mathrm{mm}), \mathrm{L}=$ comprimento da peça $(\mathrm{mm}), \mathrm{K}_{\mathrm{R}}=$ relação entre o módulo de elasticidade e espessura do suporte e esmalte, $\Delta \mathrm{C}=\mathrm{Cs}-\mathrm{Cv}$ : $\mathrm{Cs}=$ retração que o suporte sofre isoladamente desde $\mathrm{T}_{\mathrm{a}}$ até a temperatura ambiente e $\mathrm{Cv}=$ retração que o vidrado sofre isoladamente desde $\mathrm{T}_{\mathrm{a}}$ até a temperatura ambiente (\%), $\mathrm{e} \mathrm{h}=$ espessura do suporte (mm).

\section{Engobes cerâmicos}

Os engobes cerâmicos são camadas intermediárias entre o suporte e o esmalte, podem ser constituídos por argilas, fritas, caulins, zirconita, feldspatos e outras matériasprima não plásticas. Possuem várias finalidades como: opacificar o suporte cerâmico, atenuar as diferenças fisicoquímicas entre o suporte e o esmalte, diminuir a quantidade de defeitos. Como os engobes possuem composição intermediária entre a composição do suporte e do esmalte, possuem conseqüentemente, propriedades intermediárias. Pesquisadores relatam que estas propriedades se assemelham mais às propriedades do suporte cerâmico que as de um esmalte (vidro) [11]. Em consequiência do comportamento de engobes cerâmicos se distinguirem do comportamento de materiais vítreos, estes não apresentam temperatura de transição vítrea $\left(\mathrm{T}_{\mathrm{g}}\right)$. A metodologia aplicada neste trabalho visa evidenciar estas diferenças e determinar suas influências sobre a curvatura central de revestimentos cerâmicos, tendo em vista a ausência de estudos que relacionam as características dos engobes com a curvatura de revestimentos cerâmicos.

\section{Justificativa}

O crescimento da produção de revestimentos cerâmicos no Brasil aumenta a competitividade industrial fazendo com que a qualidade destes produtos esteja continuamente em evolução. Dentre os vários defeitos encontrados em revestimentos cerâmicos, a curvatura central é considerada um grave problema para a indústria cerâmica. Defeitos cerâmicos, quando presentes no consumidor final podem gerar reclamações, custos e deterioram a imagem da empresa. Visto a importância do defeito de curvatura central e sua complexidade, é de suma importância aprofundar os conhecimentos referentes à que variáveis e características das camadas que compõem o revestimento cerâmico, influenciam a curvatura central destes produtos.

\section{Objetivos e alcance da investigação}

No presente trabalho objetiva-se: apresentar os conceitos e analisar a interação suporte-esmalte e introduzir uma nova concepção do comportamento entre suporte-engobe frente às características deste conjunto; evidenciar as diferenças de comportamento entre o esmalte e o engobe cerâmico, durante a sinterização de revestimentos cerâmicos e estudar quais propriedades dos esmaltes e engobes cerâmicos influencia a curvatura central de revestimentos cerâmicos; compreender os fenômenos envolvidos na curvatura de revestimentos cerâmicos, frente à interação esmalte-engobe-suporte.

\section{MATERIAIS E MÉTODOS}

Visando alcançar os objetivos do presente trabalho, elaboraram-se quatro formulações de engobes e duas formulações de esmaltes cristalinos (transparente). Estas formulações de engobes e esmaltes visaram alcançar distintas características para possibilitar o estudo da influência destas na curvatura central de revestimentos cerâmicos. As características (variáveis) estudadas no trabalho, como já citado, foram o coeficiente de expansão térmica linear, a retração linear de queima, e a temperatura de acoplamento entre esmalte-suporte. A metodologia aplicada para avaliar a interação suporte-esmalte foi diferente da utilizada para a análise da interação suporte-engobe.

\section{Interação suporte-esmalte}

Inicialmente pesaram-se $300 \mathrm{~g}$ das formulações escolhidas em balança com precisão de $\pm 0,01 \mathrm{~g}$, a moagem foi realizada em moinho planetário por $10 \mathrm{~min}$, resultando uma suspensão com densidade entre 1850 a $1870 \mathrm{~g} / \mathrm{L}$. O resíduo de moagem foi controlado em peneira com abertura de 45 $\mu \mathrm{m}$ (ABNT \#325), permanecendo este entre 5,0 a 6,0\%. Com as suspensões dos esmaltes foram elaborados, por colagem, corpos de prova com dimensão aproximada de $1 \times 1 \times 5 \mathrm{~cm}^{3}$; respectivamente largura, espessura e comprimento. Estes foram secos em estufa a $110^{\circ} \mathrm{C}$ e sinterizados à temperatura máxima de $1050{ }^{\circ} \mathrm{C}$ em forno de queima rápida INTI FQ1300 , com velocidade de aquecimento de $70{ }^{\circ} \mathrm{C} / \mathrm{min}$ e 3 min de patamar na máxima temperatura do ciclo térmico. Posteriormente foram determinados o coeficiente de expansão térmica linear e temperatura de amolecimento em dilatômetro BP Engenharia RB $3000 \mathrm{com}$ taxa de aquecimento de 7,5 ${ }^{\circ} \mathrm{C} / \mathrm{min}$. O teste do coeficiente de expansão térmica linear do suporte, para a interação suporte-esmalte, foi realizado com o suporte previamente sinterizado nas mesmas condições das amostras de esmalte.

Tabela I - Formulações dos esmaltes utilizados nos ensaios de laboratório.

[Table I - Glaze formulations used at the laboratory tests.]

\begin{tabular}{ccc}
\hline Matérias-primas & $\begin{array}{c}\text { Esmalte } \\
\text { A }\end{array}$ & $\begin{array}{c}\text { Esmalte } \\
\text { B }\end{array}$ \\
\hline Frita A (\%) & 89,65 & --- \\
Frita B (\%) & --- & 89,65 \\
Caulim (\%) & 10,00 & 10,00 \\
Carboxi metil celulose - CMC (\%) & 0,20 & 0,20 \\
Tripolifosfato de sódio - TPF (\%) & 0,15 & 0,15 \\
\hline
\end{tabular}


As formulações dos esmaltes utilizados nesta parte do trabalho são ilustradas na Tabela I.

\section{Interação suporte-engobe}

Pesaram-se $300 \mathrm{~g}$ das formulações escolhidas em balança com precisão de $\pm 0,01 \mathrm{~g}$, a moagem foi feita em moinho planetário por $15 \mathrm{~min}$, resultando uma em suspensão com densidade entre 1850 a $1870 \mathrm{~g} / \mathrm{L}$. O resíduo de moagem foi controlado em peneira com abertura de $45 \mu \mathrm{m}$ (ABNT \#325), ficando entre 0,5 a $1,0 \%$. Com as suspensões dos engobes foram elaborados, por colagem, corpos de prova com dimensão aproximada de $1 \times 1 \times 3 \mathrm{~cm}^{3}$; respectivamente largura, espessura e comprimento. Estes por sua vez, foram secos em estufa a $110^{\circ} \mathrm{C}$ e posteriormente sinterizados na temperatura de $1050{ }^{\circ} \mathrm{C}$. Após, mediram-se as retrações de queima para cada engobe, utilizando-se quatro corpos de prova de cada engobe. Para a sinterização dos corpos de provas utilizou-se forno de queima rápida INTI FQ-1300, com velocidade de aquecimento de $70{ }^{\circ} \mathrm{C} / \mathrm{min}$ e $3 \mathrm{~min}$ de patamar na máxima temperatura do ciclo de queima. A retração linear de queima foi mensurada com o auxílio de um paquímetro. As análises dos coeficientes de expansão térmica linear dos engobes e suporte foram realizadas com auxílio de um dilatômetro BP Engenharia RB 3000, com as amostras previamente sinterizadas.

Realizou-se também, ensaios de sinterização dos engobes e suporte a verde, com o auxílio do mesmo dilatômetro. Este teste baseou-se em acompanhar as diferenças de variação dimensional durante a sinterização entre o suporte e os engobes. A taxa de aquecimento utilizada foi de $7,5^{\circ} \mathrm{C} / \mathrm{min}$ com um patamar de 3 min na temperatura máxima de $1000{ }^{\circ} \mathrm{C}$.

Para a análise da curvatura central, foram elaborados corpos de prova com suporte cerâmico de dimensão aproximada de $3 \times 0,65 \times 20 \mathrm{~cm}^{3}$; respectivamente largura, espessura e comprimento. A plicou-se sobre estes suportes uma camada de 1,2 $\mathrm{mm}$ dos esmaltes e engobes com auxílio de binil abertura 0,6 $\mathrm{mm}$. Posteriormente estes foram secos em estufa e sinterizados com o mesmo forno de queima rápida INTI FQ-1300, com igual ciclo de queima a temperatura máxima de $1050{ }^{\circ} \mathrm{C}$. Para cada engobe foram realizadas duas réplicas genuínas. Foi sinterizado também um corpo de prova

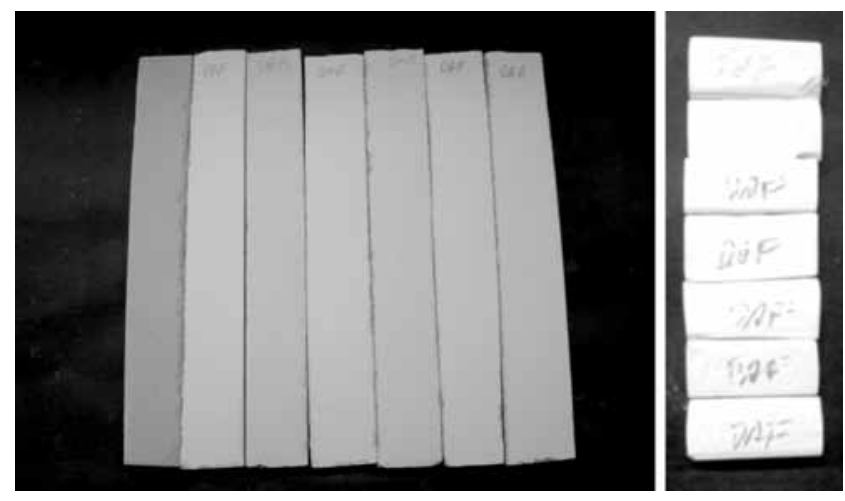

Figura 3: Corpos de prova elaborados em nível de laboratório. [Figure 3: Specimens prepared at laboratory.] sem aplicação de esmalte e engobe, visando comprovar que as curvaturas visualizadas foram provenientes da interação suporte-esmalte ou suporte-engobe, como ilustra a Fig. 3. Após a sinterização, nas determinadas temperaturas, foram mensuradas as flechas de curvatura central dos corpos de prova com o auxílio de um paquímetro.

As formulações dos engobes utilizados nesta parte do trabalho são ilustradas na Tabela II.

\section{RESULTADOS E DISCUSSÃO}

Tabela II - Formulações dos engobes utilizados nos ensaios de laboratório.

[Table II - Engobes formulations used at the laboratory tests.]

\begin{tabular}{ccccc}
\hline Matérias-prima & $\begin{array}{c}\text { Engobe } \\
\text { DBF }\end{array}$ & $\begin{array}{c}\text { Engobe } \\
\text { DBR }\end{array}$ & $\begin{array}{c}\text { Engobe } \\
\text { DAF }\end{array}$ & $\begin{array}{c}\text { Engobe } \\
\text { DAR }\end{array}$ \\
\hline Alumina (\%) & & 10,00 & & \\
Argila (\%) & 17,00 & 30,00 & 15,00 & 16,00 \\
Feldspato (\%) & 43,00 & 15,00 & 5,00 & 10,00 \\
Frita C (\%) & & & & 7,00 \\
Frita D (\%) & 12,00 & & 5,00 & \\
Frita E (\%) & & & 20,00 & 13,00 \\
Frita F (\%) & 19,00 & 26,00 & & \\
Frita G (\%) & 3,00 & & & \\
$\quad$ Nefelina & & & 10,00 & \\
Syenite (\%) & & & 34,00 & 45,00 \\
Quartzo (\%) & & 13,00 & 5,00 & 3,00 \\
Talco (\%) & & & & \\
Carboxi metil & & & 0,20 & 0,20 \\
celulose - & 0,20 & 0,20 & & \\
CMC (\%) & & & & \\
Tripolifosfato & & & & \\
de sódio - & 0,15 & 0,15 & 0,15 & 0,15 \\
TPF (\%) & & & & \\
\hline
\end{tabular}

\section{Interação suporte-esmalte}

Os resultados obtidos dos coeficientes dilatométricos dos esmaltes A, B e do suporte são ilustrados na Fig. 4 e resumidos na Tabela III. As Figs. 5 e 6 ilustram o acoplamento obtido com a análise das curvas dilatométricas entre o suporte e o Esmalte A (Fig. 5) e suporte e o Esmalte B (Fig. 6).

Nota-se que com o Esmalte $A$, ao realizarmos o acoplamento entre as curvas (Fig. 5), é observado um $\Delta c$ (diferença de contração entre suporte e esmalte) a temperatura ambiente. Com isso, aplicando a equação B (de Timoshenko) nota-se que a curvatura obtida experimentalmente é condizente com a simulada pela equação B. Ilustram-se os resultados na Tabela IV.

Para a interação entre o suporte e o Esmalte B, o resultado do acoplamento mostra que o $\Delta \mathrm{c}$ é praticamente 


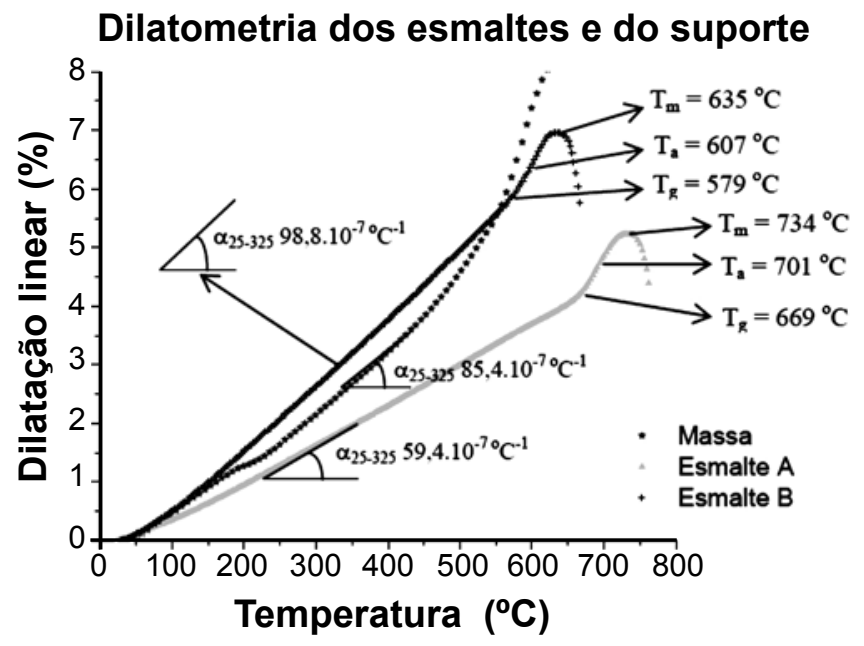

Figura 4: Resultados dilatométricos dos esmaltes e do suporte cerâmico.

[Figure 4: Expansion coefficients results of glazes and the ceramic support.]

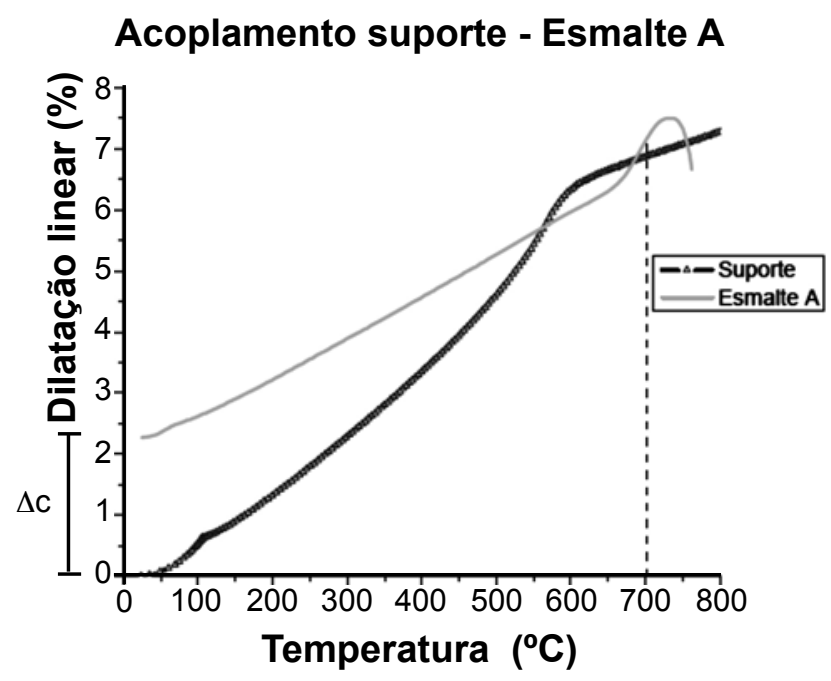

Figura 5: Acoplamento entre o suporte e o esmalte A.

[Figure 5: Coupling between ceramic support and glaze A.]

\section{Acoplamento entre suporte - Esmalte B}

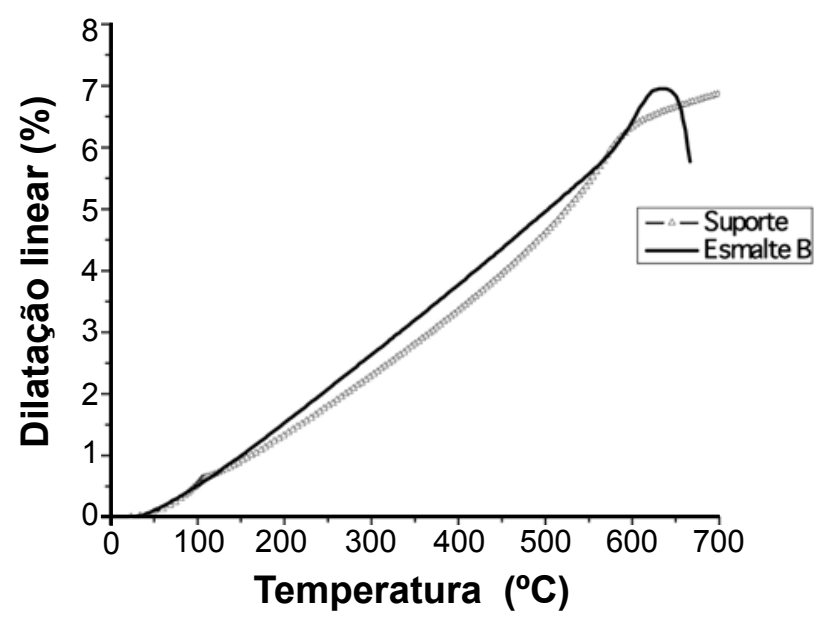

Figura 6: Acoplamento entre o suporte e o esmalte B.

[Figure 6: Coupling between ceramic support and glaze B.]
Tabela III - Caracterização dos esmaltes A, B e do suporte cerâmico.

[Table III - Characterization of the glazes A, B and the ceramic support.]

\begin{tabular}{ccccc}
\hline & $\begin{array}{c}\text { Coeficiente } \alpha_{25-325} \\
\left(.10^{-7} \mathrm{C}^{-1}\right)\end{array}$ & $\begin{array}{c}\mathrm{Tg} \\
\left({ }^{\circ} \mathrm{C}\right)\end{array}$ & $\begin{array}{c}\mathrm{Tm} \\
\left({ }^{\circ} \mathrm{C}\right)\end{array}$ & $\begin{array}{c}\mathrm{Ta} \\
\left({ }^{\circ} \mathrm{C}\right)\end{array}$ \\
\hline Esmalte A & 59,4 & 669 & 734 & 701 \\
Esmalte B & 98,8 & 579 & 635 & 607 \\
Suporte & 85,4 & --- & --- & --- \\
\hline
\end{tabular}

Tabela IV - Comparativo entre os resultados da flecha de curvatura simulados pela equação de Timoshenko e o obtido experimentalmente.

[Table IV - Comparative between results of the simulation curvature with Timoshenko equation and obtained experimentally.]

\begin{tabular}{ccc}
\hline $\begin{array}{c}\text { Interação entre } \\
\text { Suporte - }\end{array}$ & $\begin{array}{c}\mathrm{D}(\mathrm{mm}) \\
\text { Timoshenko }\end{array}$ & $\begin{array}{c}\mathrm{D}(\mathrm{mm}) \\
\text { Experimental }\end{array}$ \\
\hline Esmalte A & 1,5 & 2,0 \\
Esmalte B & 0,0 & 0,0 \\
\hline
\end{tabular}

nulo (Fig. 6), portanto a flecha de curvatura, pela equação de Timoshenko, deveria também ser nula, como obtido experimentalmente, como ilustrado na Tabela IV.

\section{Interação Suporte-Engobe}

A Tabela $\mathrm{V}$ mostra o planejamento fatorial $2^{2}$ utilizado na pesquisa para avaliar a influência dos fatores: coeficiente de expansão térmica linear (dilatação) e diferença de retração linear entre engobe e suporte, estes sinterizados a $1050{ }^{\circ} \mathrm{C}$, como mencionado anteriormente.

Tabela V - Planejamento fatorial $2^{2}$ utilizado para avaliar a influência das características do engobe sobre a curvatura central.

[Table V-Factorial design $2^{2}$ used to evaluate the influence of the engobe characteristics on the curvature.]

\begin{tabular}{lcc}
\hline Fator $\quad$ Nível & -1 & +1 \\
\hline Dilatação $\left(.10^{-70} \mathrm{C}^{-1}\right)$ & 55 & 90 \\
$\begin{array}{c}\text { Diferença da Retração } \\
\text { Engobe-Suporte (\%) }\end{array}$ & 0,95 & 2,92 \\
\hline
\end{tabular}

A Tabela VI mostra os resultados do planejamento experimental realizado com os engobes. As denotações desta tabela referem-se: DBR engobe com dilatação baixa refratário; DAR engobe com dilatação alta refratário; DBF engobe com dilatação baixa fundente e DAF engobe com dilatação alta fundente.

A Fig. 7 relata uma acentuada diferença de retração linear, provocada pela sinterização, entre o suporte (massa) e o engobe DAR. Experimentalmente a flecha de curvatura 
Tabela VI - Resultados das flechas de curvatura obtidas a partir do planejamento fatorial $2^{2}$ utilizado.

[Table VI - Curvature results obtained by factorial design $2^{2}$.]

\begin{tabular}{cccc}
\hline ENGOBE & $\begin{array}{c}\text { Dilatação } \\
\left(.10^{-7} \mathrm{C}^{-1}\right)\end{array}$ & $\begin{array}{c}\text { Diferença Retração } \\
\text { Suporte-Engobe } \\
(\%)\end{array}$ & $\begin{array}{c}\text { Curvatura } \\
(\mathrm{mm})\end{array}$ \\
\hline DBR & 55 & 2,92 & 6,45 \\
DAR & 90 & 2,92 & 5,30 \\
DBF & 55 & 0,95 & 2,86 \\
DAF & 90 & 0,95 & 1,30 \\
\hline
\end{tabular}

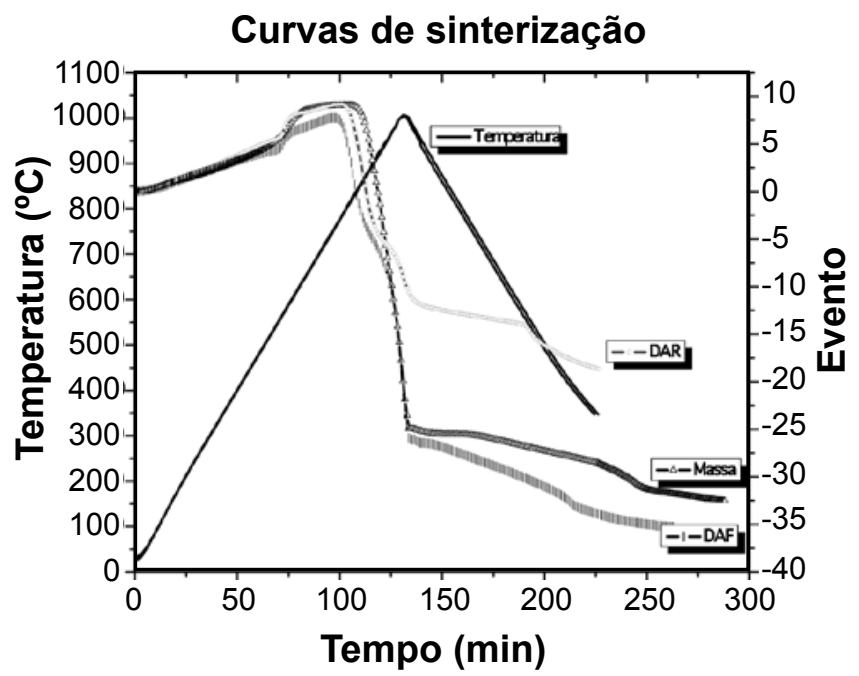

Figura 7: Curvas de sinterização dos engobes com dilatação alta e o suporte.

[Figure 7: High thermal expansion engobes and ceramic support sintering curves.] para este engobe foi de $5,30 \mathrm{~mm}$; enquanto para o engobe DAF a flecha de curvatura experimental obtida foi somente de $1,30 \mathrm{~mm}$.

A Fig. 8 mostra o comportamento dos engobes com dilatação baixa $\left(55.10^{-7} \mathrm{C}^{-1}\right)$ frente ao suporte. Analisando-a nota-se que o engobe DBF apresentou uma sinterização muito similar à sinterização do suporte cerâmico, com isso a diferença de retração linear entre ambos foi praticamente nula, levando a uma curvatura não muito acentuada. Para o engobe refratário (DBR) nota-se uma diferença de retração alta entre este e o suporte cerâmico, após a sinterização. Experimentalmente observou-se que o engobe DBR, que

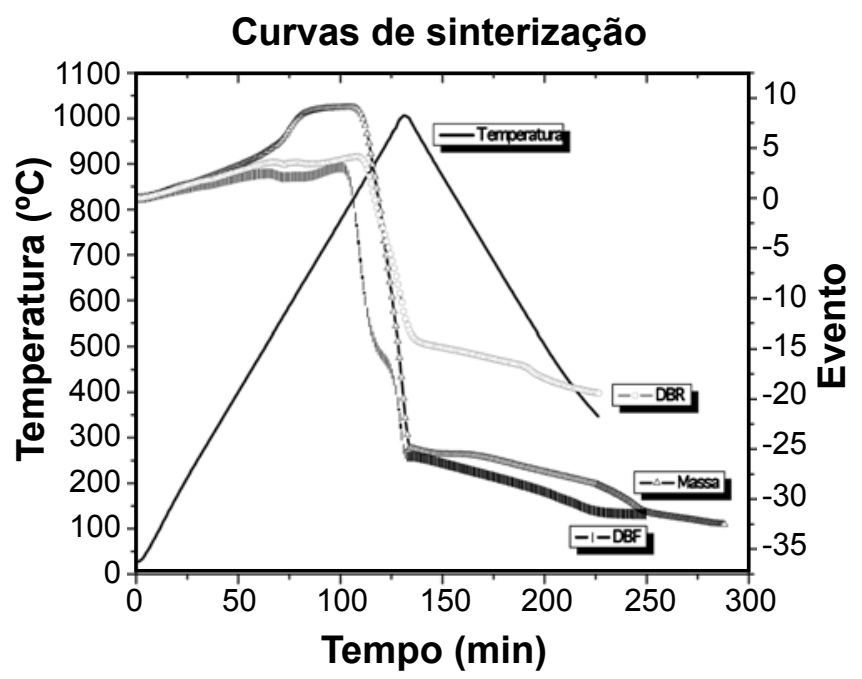

Figura 8: Curvas de sinterização dos engobes com dilatação baixa e o suporte.

[Figure 8: Low thermal expansion engobes and ceramic support sintering curves.]

Tabela VII - Tabela da ANOVA obtida com os resultados.

[Table VII - Table of ANOVA obtained with the results.]

\begin{tabular}{cccccc}
\hline Fator & $\begin{array}{c}\text { Soma dos } \\
\text { Quadrados } \\
(\mathrm{SS})\end{array}$ & $\begin{array}{c}\text { Grau de } \\
\text { Liberdade } \\
(\mathrm{df})\end{array}$ & $\begin{array}{c}\text { Média dos } \\
\text { Quadrados } \\
(\mathrm{MS})\end{array}$ & Fator F & Valor p \\
\hline Coef. Exp. Térmica & 1,836 & 1 & 1,836 & 43,6889 & 0,09559 \\
Diferença Retração & 14,402 & 1 & 14,402 & 342,7014 & 0,03435 \\
Erro & 0,042 & 1 & 0,042 & & \\
Total SS & 16,280 & 3 & & & \\
\hline
\end{tabular}

Tabela VIII - Tabela da ANOVA, estimativas de efeitos obtidas com os resultados.

[Table VIII - Table of ANOVA, effect estimates obtained with the results.]

\begin{tabular}{ccccc}
\hline Fator & Coeficiente & $\begin{array}{c}\text { STD erro } \\
\text { coeficiente }\end{array}$ & $\begin{array}{c}-95 \% \\
\text { Limite Confiança }\end{array}$ & $\begin{array}{c}+95 \% \\
\text { Limite Confiança }\end{array}$ \\
\hline Média / Intersecção & 3,9775 & 0,1025 & 2,675 & 5,279 \\
Coef. Exp. Térmica & $-0,6775$ & 0,1025 & $-1,979$ & 0,625 \\
Diferença Retração & 1,8975 & 0,1025 & 0,595 & 3,199 \\
Total SS & 16,280 & 3 & & \\
\hline
\end{tabular}


apresentou diferença de retração com o suporte, teve flecha de curvatura acentuada, resultados estes ilustrados na Tabela VI.

A análise estatística ilustrada na Tabela VII, mostra a tabela da ANOVA onde temos os resultados de significância para os fatores (variáveis) "coeficiente de expansão térmica linear" e "diferença de retração entre suporte e engobe". Analisando-a notamos que a variável "diferença de retração entre suporte e engobe" foi o fator significante, pois o valor de "p" ficou abaixo de 5\%, sendo que a dilatação linear não alterou a curvatura central, dentro da faixa estudada.

A Tabela VIII ilustra a estimativa de efeitos da análise estatística. Com esta pode-se obter os coeficientes para a regressão linear onde relacionamos a flecha de curvatura (D) com as variáveis estudadas.

De acordo com a estimativa de efeitos a regressão linear com melhor ajuste para os resultados experimentais se expressa matematicamente de acordo com a equação $\mathrm{C}$ :

$$
\mathrm{D}=3,9775+1,8975 \text {. (Diferença retracão) }
$$

Como a variável "coeficiente de expansão térmica" mostrou-se sem significância para a flecha de curvatura central, esta pode ser desprezada na equação $\mathrm{C}$.

Pareto Chart of Standardized Effects; Variable: Curvatura (mm) $2^{\star \star}(2-0)$ design; MS Residual $=0,42025$ DV: Curvatura (mm)

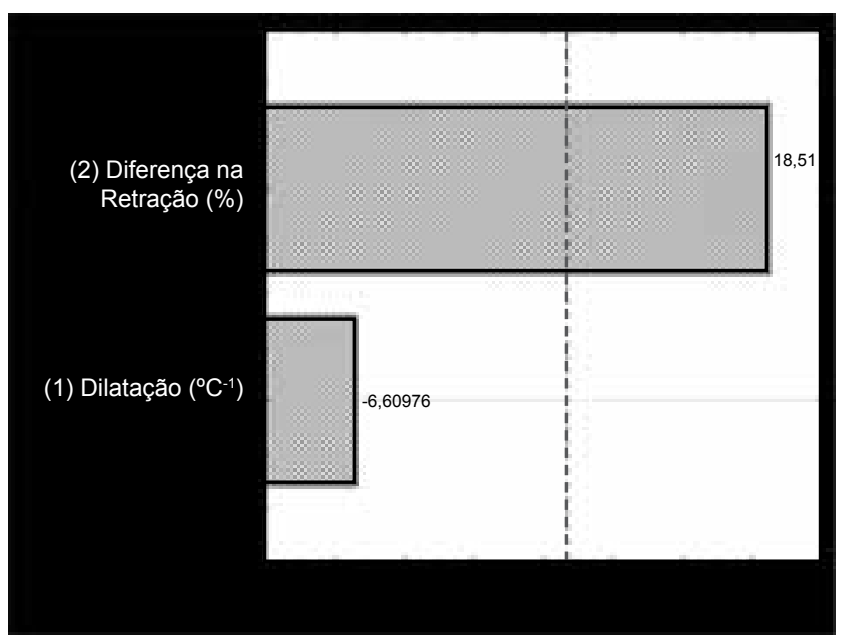

Figura 9: Gráfico de Pareto, ilustrando a significância das variáveis estudadas frente a curvatura central.

[Figure 9: Pareto graphic, shows the significance of the variables studied.]

Com o gráfico de Pareto, ilustrado na Fig. 9, podemos afirmar com $95 \%$ de certeza, que a variável significante é a "diferença de retração linear entre o suporte-engobe" e que o coeficiente de expansão térmica linear (dilatação) não se mostrou significante para a faixa estudada entre 55 a $90 \cdot 10^{-7} \mathrm{C}^{-1}$.

\section{CONSIDERAÇÕES FINAIS}

De acordo com os resultados encontrados neste trabalho, evidenciou-se que o comportamento de esmaltes e engobes, durante a sinterização de produtos cerâmicos, é muito distinto. Visto que os esmaltes cerâmicos brilhantes possuem um comportamento similar ao de um vidro; portanto, para temperaturas acima da temperatura de amolecimento, estes não exercem influência (força/tensão) sobre o suporte cerâmico [9]. Os esmaltes vem então a exercer tensão somente durante o processo de resfriamento de revestimentos cerâmicos, após a sinterização. Para estes casos a equação de Timoshenko, que leva em conta somente a parte elástica do comportamento mecânico de materiais cerâmicos, é muito bem aplicada. Portanto, com os resultados obtidos experimentalmente, podemos estabelecer que para a interação suporte-esmalte a análise do coeficiente de expansão térmica linear (dilatação térmica) é de fundamental importância para descrever a curvatura central de revestimentos cerâmicos.

Evidenciou-se que para os engobes cerâmicos estudados, não se observou temperatura de amolecimento, vide Figs. 7 e 8, dentro das temperaturas empregadas na sinterização. Portanto estes exerceram força e/ou tensão sobre o suporte cerâmico quando a "diferença de retração linear" de queima entre estes foram acentuadas. Portanto, os resultados mostram que para a interação suporte-engobe, a variável "diferença de retração linear" mostrou-se significativamente importante para avaliarmos a curvatura central de revestimentos cerâmicos, enquanto o coeficiente de expansão térmica linear mostrou-se sem significância, com $95 \%$ de certeza. Os resultados experimentais são válidos somente para os engobes e o suporte estudado nesta pesquisa.

\section{AGRADECIMENTOS}

À Endeka Ceramics pela cessão das matérias-primas, ao LaRC-UFSCar pela disponibilidade dos equipamentos e à CAPES pelo auxílio financeiro.

\section{REFERÊNCIAS}

[1] S. Giménez, G. Beltrán, S. Horrillo, G. Mallol, V. Cantavella, M. J. Daroca, Cerâmica Industrial 5, 3 (2000) 10.

[2] V. Cantavella, Tesis Doctoral, Departamento de Ingeniería Química, Universidad Jaume I de Castellón, Castellón, Espanha (1998).

[3] G. Mallol, D. Llorens, G. Beltrán, Técnica Cerámica 276 (1999) 855.

[4] L. Lorici, Cerámica y Cristal 114 (1995) 19.

[5] Autori vari, Tecnología de la Fabricación de Azulejos, Ed. Litografía Castellón S.A., Castellón, Espanha (1990) $\mathrm{X}-25$.

[6] M. Monzó, Tesis Doctoral, Departamento de Ingeniería Química, Universidad Jaume I de Castellón, Castellón, Espanha (1994).

[7] J. L. Amorós, V. Beltrán, A. Blasco, Defectos de fabricación de pavimentos y revestimientos cerámicos, 
Castellón, Espanha, Asociación de Investigación de las Industrias Cerámicas (1991) 159.

[8] A. Escardino, J. L. Amorós, F. Negre, Influencia de las variables de proceso, Taulells 3 (1985) 3.

[9] J. L. Amorós, F. Negre, A. Belda, Técnica Cerámica 178 (1989) 582.

[10] J. L. Amorós, A. Blasco, J. V. Carceller, Técnica Cerámica 179 (1989) 644.

[11] G. R. Santos, F. G. Melchiades, A. O. Boschi, Ceram.
Ind. 12, 5 (2007) 22.

[12] M. Paganelli, C. Venturelli, Ceram. Forum Int. 86, 6 (2009) 37.

[13] S. Pracidelli, Ceram. Ind. 13, 1/2 (2008) 8.

[14] W. D. Callister Jr., Materials Science and Engineering: an Introduction, $7^{\text {th }}$ Ed., John Wiley \& Sons, Inc., New York, NY, EUA (2007) p.472.

[15] S. B. Yamaki, A. G. Pedroso, T. D. Z. Atvars, Quim. Nova 25, 2 (2002) 330.

(Rec. 01/10/2010, Ac. 25/03/2011) 